\title{
Particle Tracking Velocimetry and Accelerometry (PTVA) measurements applied to quasi-two-dimensional multi-scale flows
}

Received: date / Accepted: date

\begin{abstract}
We have developed and validated a new adaptive method, Particle Tracking Velocimetry and Accelerom etry (PTVA), to measure velocity and acceleration from the post-processing of Particle Pracking (PT) data. This method is shown to be more accurate than non-adaptive methods based on PT: errors are about 6 times smaller on velocity measurements and about 4 times smaller on acceleration ones. We apply this method to a turbulentlike flow generated and controlled in the laboratory. Taking advantage of the Eulerian repeatability of our multiscale laminar flow, we are able to extract the acceleration field, a, and all terms of Navier-Stokes equation. To complete this we extract $\mathbf{u} \cdot \mathbf{a}$ and $\nabla \cdot \mathbf{a}$ fields. We finally compare the Probability Density Function of the acceleration components of our turbulent-like flow with the one of highly turbulent flows and show that they are similar.

The quality of these PTVA results and their robustness (in particular to local convection) are extremely encouraging. This method allows access to a deeper insight into the physic of turbulent-like flows and its high accuracy may apply to a broader range of flows.
\end{abstract}

Keywords Lagrangian measurements · Acceleration . Quasi-two dimensional multi-scale flows · Laboratory experiments

\section{Introduction}

Lagrangian statistics are important for transport, stirring and mixing. The Lagrangian acceleration, $\mathbf{a}$, is at the very base of fluid motion (momentum equation) whilst it is a non trivial flow component, e.g. Tsinober (2001a),

\section{Rossi}

Department of Aeronautics, Imperial College London, Prince Consort Rd, London SW7 2AZ, United Kingdom

E-mail: 1.rossi@imperial.ac.uk

\section{S. Ferrari}

Dipartimento di Ingegneria del Territorio, Università degli Studi di Cagliari, Piazza d'Armi, 09123 Cagliari, Italy neither it is trivial to measure. As a matter of fact, if a certain number of authors have studied, via numerical simulations, acceleration properties, e.g.: Vedula and Yeung (1999), Tsinober et al. (2001b), Biferale et al. (2004), Goto et al. (2005); and its relation to inertial particle clustering, e.g. Chen et al. (2006), very few examples of experimental measurements are available in the literature.

To model and study some of the multiple-scale aspects associated to turbulence but also to stirring and mixing, Rossi et al. (2005, 2006a and 2006b) generate and control a quasi-two dimensional multiple-scale flow in the laboratory and numerical simulations. This flow has a multiple-scale distribution of stagnation points according to the flow structures they are connected to. One advantageous property of the present flow is that topology and temporal evolution can be easily controlled.

We want to measure accurately velocity and acceleration from PT on this multiple-scale flow. The measurement needs to be accurate at all the flow scales, with a critical insight given to the close vicinity of points where the velocities are small but with strong curvature of the streamlines and high strain (e.g. hyperbolic stagnation points). In addition, for particles dispersion (and so stirring and mixing), it is not only a which is important but also its divergence, $\nabla \cdot \mathbf{a}$ (Vassilicos (2002)). The estimation of $\nabla \cdot \mathbf{a}$ needs accuracy on a and on the spatial derivatives of $\mathbf{a}$. We thus want the acceleration measurement to be accurate at large and small flow length-scales, including large and small intensities of the acceleration.

As the Lagrangian acceleration can be split into two Eulerian components: a local and a convective accelerations (equation (1), where the velocity $\mathbf{U}_{c}$ represents a Galilean reference); some authors have measured it in Eulerian frames of reference (e.g.: Christensen and Adrian (2002), Dong et al. (2001), Lowe and Simpson $(2005))$.

$\mathbf{a}=\frac{D \mathbf{u}}{D t}=\underbrace{\frac{\partial \mathbf{u}}{\partial t}+\left(\mathbf{U}_{c} \cdot \nabla\right) \mathbf{u}}_{\text {local acceleration }} \underbrace{-\left(\mathbf{U}_{c} \cdot \nabla\right) \mathbf{u}+(\mathbf{u} \cdot \nabla) \mathbf{u}}_{\text {convective acceleration }}$ 
Despite the Galilean invariance of Lagrangian acceleration, local and convective acceleration are not. If $\left(\mathbf{U}_{c}\right.$. $\nabla) \mathbf{u}$ is dominant, the Lagrangian acceleration becomes relatively weak but is not strictly zero. This point is crucial for Eulerian measurements, as they need to be of high precision to measure weak "fluctuations" of acceleration compared to $\left(\mathbf{U}_{c} \cdot \nabla\right) \mathbf{u}$ for both local and convective accelerations. Particle Tracking Velocimetry (PTV) techniques, where particles are tracked one by one, are amenable to measure Lagrangian acceleration.

One of the main issues with PTV is its sensitivity to noise. In order to reduce it, Virant and Dracos (1997) computed the velocity using the displacement of particles over more than two consecutive frames. More recently, low-pass filters have been used on the trajectories to reduce the noise. They consist in trajectory approximation using moving polynomials of various order (parabola in La Porta et al. (2001) and in Voth et al. (2002), third order polynomial in Luthi et al. (2005)) and filtering kernel (Mordant et al. (2004)). These techniques imply to choose a time window of constant duration (i.e., the number of consecutive positions used to fit the polynomial), on the basis of a particular data set.

Due to the multiple-scale nature of our flow, it is not possible to define a fixed number of positions which could work for all length/time-scales. A large number of positions would efficiently remove the noise at large scales but would delete small scale fluctuations, whereas a small number of positions would keep small length/time-scales fluctuations emphasizing noise at the large length/timescales.

We propose a method which uses an adaptive number of positions (respectively time window) for the trajectory approximation depending on the local flow properties. This method holds the potential to go beyond the measurement of local convection so as to extract more accurately the acceleration and velocity. The method is validated using an analytical approach and its performance is then assessed. We then compare this adaptive method to methods using a fixed number of positions.

Once the method has been validated, we measure the velocity and acceleration from our multiple-scale flow experiments. Taking advantage of the Eulerian repeatability of our multiple-scale laminar flows, we study the multiple-scale distribution of $\mathbf{u}$ and $\mathbf{a}$ (according to the flow geometry and its fractal forcing) and compute $\mathbf{u} \cdot \mathbf{a}$, $\nu \nabla^{2} \mathbf{u}$ and $\nabla \cdot \mathbf{a}$ from these fields. Finally, this study is completed with the PDF of Lagrangian acceleration obtained in our flow.

\section{Particle Tracking Velocimetry and Accelerometry, PTVA, method}

The PTVA method is based on an adaptive local polynomial approximation of order $\mathrm{n}(n>1)$ to estimate the trajectories. To simplify the writing in this paper, we solve it using vectors $(\mathbf{x}, \mathbf{u}, \ldots)$. Results and validations presented in this paper use this vectorial form. For extra accuracy on each coordinate (but with extra time of computation) it can easily be adapted for each separate coordinate $\left(\mathrm{x}, u_{x}, \mathrm{y}, u_{y}, \ldots\right)$.

\subsection{Basics and trajectory approximation}

The data input of the method consists in tracked particles' positions in time, $\mathbf{x}(\mathbf{t})^{1}$. In our experiments (section 4) we use two PTV codes: DigiFlow, Dalziel (1992), and GPTV, Querzoli (1996), to extract the particle positions. Although different on many aspects, they can both extract the successive positions occupied by a particle, following these steps: 1) identification of the particle centroids, after one or more thresholds have been applied on the picture (representing a collection of bright particle over a dark background); 2) trajectory recognition by linking the particles' positions at time $t+1$ with those at time t, matching various criteria (see Dalziel (1992) and Querzoli (1996) for more details).

In the PTVA method, trajectories are approximated locally as $\mathbf{x}_{n}(t)=\mathbf{b}_{n} t^{n}+\ldots+\mathbf{b}_{1} t+\mathbf{b}_{0}$. The velocity and acceleration are extracted from the trajectory $\mathbf{x}_{n}(t)$ with the time derivatives: $\mathbf{u}_{n}(t)=n \mathbf{b}_{n} t^{n-1}+\ldots+\mathbf{b}_{1}$ and $\mathbf{a}_{n}(t)=n(n-1) \mathbf{b}_{n} t^{n-2}+\ldots+2 \mathbf{b}_{2}$. If the acceleration is time dependent, the order of interpolation should be larger than 2 or the sampling of the experiment should be performed at very high frequencies to obtain a minimum of accuracy.

The time and length-scale resolutions are important to properly measure the velocity and acceleration as they rely on two successive time derivatives of the optical particle tracking (pixel based measure of displacement). To illustrate the relation between the spatial and temporal resolutions, we use the concept of a convected eddy. Its diameter (and perimeter) refers to a characteristic length-scale and its turn-over time refers to a characteristic time-scale. The row "line a" of figure 1 illustrates an analytical trajectory related to the same convected eddy with different frequencies and duration of tracking. The first column corresponds to a reference acquisition during one turn-over time. The second column corresponds to an acquisition with a higher frequency during the same period of time. The third column corresponds to an acquisition with a lower frequency and with the same number of positions. As a consequence the eddy is tracked (line a) during two turn-over times illustrated by two different colors. In line $\mathrm{b}$ and $\mathrm{c}$ the convection is removed. Line $\mathrm{b}$ illustrates the use of a constant number of positions for the approximation. Line $\mathrm{c}$ illustrates the use of an adaptive number of positions so as to perform the approximation during one turn-over time. The second column illustrates the importance to adjust the

1 The PTVA method is based on a post-processing algorithm using existing tracked particle positions. The tracking of the particles is performed separately. 

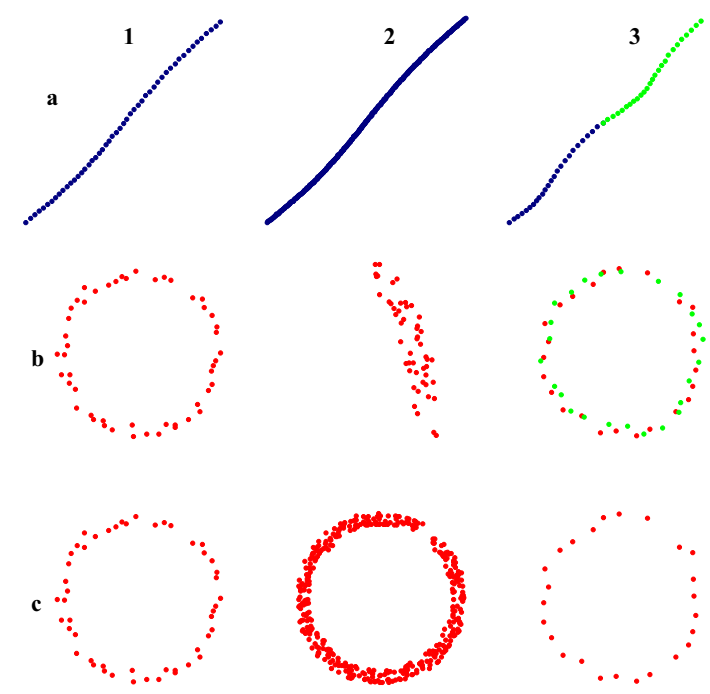

Fig. 1 Line a shows tracked positions of a particle within a convected eddy at various frequencies. The convection is removed in lines b and c. Line $b$ corresponds to the use of a constant number of positions, line $c$ corresponds to an adaptive number of positions systematically catching one turn over time of the convected eddy. Columns correspond to different frequency sampling: (1) one turn over time, reference frequency; (2) one turn-over time, higher frequency; (3) two turn-over time, lower frequency.

time window used to approximate the trajectory according to the level of noise when local convection has been removed.

In addition, it is important to notice that the order, $\mathrm{n}$, of the trajectory's approximation determines the maximum number of changes, $n-1$, in velocity sign in the local convective frame. This gives a limitation in the number of loops (a loop corresponds to 3 zero crossings for the coordinates values in the local convective frame) which could be measured, e.g. half a loop for an order 2, 1.5 loops for an order 4 . If the time window is too large, e.g. figure 1 column 3 line $\mathrm{b}$ for $n<5$, the approximation is incorrect.

Considering a flow's time-scale to be measured, $T_{\text {flow }}$, and the duration of the time-window, $N \Delta t(N$ is the number of time steps $\Delta t$ used for the approximation), to properly extract a structure turn-over time we should have $N \Delta t<\frac{n-1}{2} T_{\text {flow }}$. The corresponding tracking frequency, $f=1 / \Delta t$, is $f>\frac{N}{n-1} \frac{2}{T_{\text {flow }}}$. If $T_{\text {small }}$ is the smallest flow's characteristic time-scale (to be measured), the frequency of the camera is initially adjusted so as to correctly sample this time-scale. Inversely, if the frequency of acquisition is imposed, the smallest time-scale that could be extracted by the considered polynomial approximation is given by $T_{\text {small }}>\frac{N}{n-1} \frac{2}{f}$.

In terms of length-scale, the length $\Delta$ that the polynomial approximation is able to extract should be kept smaller than the displacement related to the smallest flow structure. Using the picture of a convected eddy (figure 1) this is estimated by $\Delta \leq \Delta_{\max }=\pi \phi_{e d} \frac{n-1}{2}+$ $\Delta_{\text {conv }}$, where $\Delta_{\text {conv }}$ is the length-scale corresponding to the average convection during tracking, $\phi_{e d}$ is the diameter of the smallest eddy to be tracked and $n$ the order of the polynomial approximation.

It should be noticed that an order of interpolation higher than $n=2$ allows larger displacements and timewindows whilst correctly approximating the smallest length and time scales. For practical considerations, this could permit to improve small scales' resolution (length and time) for a given frequency of acquisition and/or to use a lower camera frequency for given small flow scales (time and space).

To adapt the method to the local flow properties, $\Delta_{\text {conv }}$ is associated with the summation of $\overline{\mathbf{u}} \Delta t$, where $\overline{\mathbf{u}}$ is the local mean Lagrangian velocity. The remaining part of the displacement is then associated to a flow structure which could either be a convected eddy, as in figure 1, or any "temporal structure" due to various time dependencies. The proposed algorithm does not rely on the existence of eddies.

Finally, the considered adaptive method relies only on physical criteria related to the flow length/time-scales to be extracted, i.e the smallest turn-over time-scale of the flow and the corresponding length-scale. The method attempts to extract the information related to one turnover time of this given "flow structure" (and/or scale) in the corresponding Lagrangian frame. To do that, we choose an order 4 for the polynomial approximation of trajectories which provides an order 2 for the acceleration polynomial. This is one order larger than the minimum required to approximate one turn-over time (3 zero crossings).

Once the sampling of the smallest turn-over timescale is adapted via the frequency of the camera, the small length-scale targeted, $\Delta_{\text {target }}$, becomes the main input parameter of the computing method. The smallest length-scale's diameter, $\phi_{\text {small }}$, that the method $(n=4)$ could accurately extract is $\phi_{\text {small }}=\frac{2 \Delta_{\text {target }}}{3 \pi}{ }^{2}$

\subsection{A two stages acceleration measurement}

The PTVA algorithm which lies on an acceleration measurement in two stages is now discussed. It should be noticed that the two stages do not imply a double filtering of the trajectories. Both stages take as input the particle positions extracted via PTV.

In the first stage, a first approximation is performed to have a measurement of the velocity in each position that can be used in the second stage to remove the local convection velocity. To be accurate at small and large velocities, we adapt the number of positions, $N_{t}$, used

\footnotetext{
2 If $\phi_{\text {small }}$ is unknown, as a first iteration, the initial $\phi_{\text {small }}$ could be estimated via the resolution of the measurement (e.g. \pm 1 pix) and the diameter of the particles so as to determine $\Delta_{\text {target }}$ (taking $\Delta_{\min }=\frac{\phi_{\text {small }}}{2}$ and $\Delta_{\max }=2 \Delta_{\text {target }}$ ). The measurements and post-processing can then be adjusted according to this first iteration.
} 
for the trajectories approximation. The displacement $\Delta$ is given by equation (2):

$$
\Delta=\sum_{i=-N_{t} / 2}^{N_{t} / 2}\left\|\mathbf{x}_{\mathbf{i}+\mathbf{1}}-\mathbf{x}_{\mathbf{i}}\right\|
$$

Considering the minimum displacement, $\Delta_{\text {min }}$, needed to have a signal versus noise ratio high enough (if $\sigma_{\text {noise }}$ is the typical noise of the position tracking, we should have $\Delta_{\min } \gg \sigma_{\text {noise }}$ ), $N_{t}$ is adapted so as to have $\Delta_{\min } \leq \Delta \leq \Delta_{\max }$ and $N_{\min } \leq N_{t} \leq N_{\max } . \Delta_{\max }$ is the displacement associated with the small scales to be measured which includes convection. $N_{\min }$ is the minimum number of positions required for approximation, e.g. $N_{\min }=2 n-1$. $N_{\max } \Delta t$ should be longer than the longest time-scale to measure, e.g. large-scale turn-over time. We then measure a first velocity via time derivative of the local polynomial approximation performed with this latest $N_{t}$.

In the second stage, we perform a new approximation of the trajectories using the Lagrangian velocity measured in the previous stage to remove the local convection. For this, we use the local (and adaptive) time average of the Lagrangian velocity of the tracked particle, $\overline{\mathbf{u}}$ given by equation (3):

$\overline{\mathbf{u}}=\frac{1}{N_{t}} \sum_{i=-N_{t} / 2}^{N_{t} / 2} \mathbf{u}_{i}$

This Lagrangian average velocity enables to compute the convected displacement, $\Delta_{s}$, defined by equation (4).

$\Delta_{s}=\sum_{i=-N_{t} / 2}^{N_{t} / 2}\left\|\mathbf{x}_{i+1}-\mathbf{x}_{i}-\overline{\mathbf{u}} \Delta t\right\|$

Finally, once the local convection has been removed via $-\overline{\mathbf{u}} \Delta t$ in equation (4), the algorithm self-adapts ${ }^{3}$ to get close to one turn-over of the smallest scales to be extracted, $\Delta_{s} \cong \Delta_{\text {target }}$. $N_{t}$ is still bounded between $N_{\min }$ and $N_{\max }$. The values of $N_{\min }$ and $N_{\max }$ used in the experiments are given in section 4.2. This can be achieved with polynomial functions of order larger than 3. In section 6 we use polynomial functions of order 4 .

The velocity and acceleration are then measured via time derivatives of the local polynomial approximation performed with this last $N_{t}$.

The novelty of the method comes from its adaptability and its mean Lagrangian velocity correction.

\subsection{Noise and feedback on measurements}

A least mean square method is used for the polynomial approximations. This provides for each approximation

\footnotetext{
${ }^{3}$ Starting from an initial value of $N_{t}$ (keeping the value of $N_{t}$ used in the previous time step for a same trajectory), if $\Delta_{s}<\Delta_{\text {target }}$ then $N_{t}$ is increased (else $N_{t}$ is decreased), until $\left|\Delta_{s}-\Delta_{\text {target }}\right| \leq \epsilon$, with $\epsilon$ small compared to $\Delta_{\text {target }}$.
}

the standard deviation, $\sigma_{\text {noise }}$. The ratio $\sigma_{\text {noise }} / \Delta_{s}$ gives a good feedback on the quality of the acceleration measurement (with $N_{t} \sigma_{\text {noise }} / \Delta_{s}<<1$ when $\mathbf{a} \neq \mathbf{0}$ ). We will later use this function to characterize (and validate) the quality of the acceleration measurement according to the noise level.

In the present description of the PTVA method, we consider that the total noise level $N_{t} \sigma_{\text {noise }}$ is low compared to the useful displacement $\Delta_{s}$. This might not be the case with high level of noise (high values of $\sigma_{\text {noise }}$ ) and/or with a large frequency over-sampling (high values of $\left.N_{t} \sigma_{\text {noise }}\right)$. In this case, an additional correcting term $\left(-N_{t} \sigma_{\text {noise }}\right)$ should be included in equation (4) giving $\Delta_{s}=\sum_{i=-N_{t} / 2}^{N_{t} / 2}\left\|\mathbf{x}_{i+1}-\mathbf{x}_{i}-\overline{\mathbf{u}} \Delta t-N_{t} \sigma_{\text {noise }}\right\|$. The drawback, in this case, is that the method will often naturally tends towards the maximum number of positions allowed for the approximation. The measure then becomes more a problem of noise filtering.

The experiments presented in this paper are highly resolved spatially with a moderate frequency of the camera combined with a low level of noise. Hence they do not require this correction and then benefit fully from the PTVA method.

\section{Validation and measurement accuracy}

\subsection{Analytical approach}

We choose a set of geometrical structures analytically defined, from which the exact velocity and acceleration are known in each point. We then consider the measurement accuracy for a broad range of flow structures with and without convection term. These geometrical trajectories can be considered as being characteristic sections of real flow trajectories. Their velocity intensity is adjusted so as to have similar maximum velocity between these geometrical trajectories and the ones from experiments. For each set we perform computation over more than 1000 trajectories and more than $210^{6}$ positions.

\subsubsection{Choice of convected geometrical structures}

$$
\left\{\begin{array}{l}
u_{o t}=G\left(y-y_{0}\right) \\
v_{o t}=K G\left(x-x_{0}\right)
\end{array}\right.
$$

Equation (5) gives different velocity fields $\left(u_{o t}, v_{o t}\right)$ according to the values of $\mathrm{G}$ and $\mathrm{K}(-1 \leq K \leq 1)$. In particular, $-1 \leq K<0$ corresponds to a velocity field with an elliptical stagnation point, $K=0$ to an unidirectional shear flow and $0<K \leq 1$ to a velocity field with a hyperbolic stagnation point, Ottino (1989). $x_{0}$ and $y_{0}$ are the centres of the geometrical structures considered and correspond to the positions of the stagnation points.

The Galilean transformations of these velocity fields are obtained in equation (6), where $-\mathbf{U}_{\mathbf{c}}$ is the convec- 
tive velocity (coordinates $-U_{c_{x}}$ and $-U_{c_{y}}$ ) of flow structures and $\mathrm{t}$ is the time.

$\left\{\begin{array}{l}u=u_{o t}+G U_{c_{y}} t-U_{c_{x}} \\ v=v_{o t}+K G U_{c_{x}} t-U_{c_{y}}\end{array}\right.$

At time $\mathrm{t}=0$, the stagnation point of the convected structure is at the position $\left(x_{0}, y_{0}\right)$ in the observer frame. This reference point is moving at $-\mathbf{U}_{\mathbf{c}}$ in the observer frame. To quantify the convection according to the intensity of the convected flow structures, we consider $\mathbf{u}=$ $-\mathbf{U}_{\mathbf{c}}+\mathbf{u}^{\prime}$. We thus define the convection coefficient by $C_{\text {conv }}=\mathbf{U}_{\mathbf{c}} / \mathbf{u}^{\prime}{ }_{\text {rms }}$, where $\mathbf{u}^{\prime}{ }_{\text {rms }}$ is the root mean square of $\mathbf{u}^{\prime}$. When $C_{\text {conv }}=0$ there is no convection. When $C_{c o n v}=1$ the convection is comparable to the velocity intensity, increasing the difficulty of the acceleration measurements, equation (1). Later on, we study the quality of the acceleration measurement according to different values of $C_{\text {conv }}$.

\subsubsection{Accuracy of the PTVA method}

To analyse the sensibility of the measurement to the noise, we add a controlled noise to the analytical trajectories. Two different noises are used: the first one is a Gaussian noise with selected standard deviation, the second one is a directional noise of selected amplitude. The latter is defined, point by point, by an angle and a distance, both randomly generated following an uniform distribution (bounded between 0 and $2 \pi$ for the angle and between 0 and a selected amplitude). The values of the noise intensity $(0 \mathrm{px}, 0.03 \mathrm{px}, 0.06 \mathrm{px}, 0.1 \mathrm{px}, 0.2$ $\mathrm{px}, 0.3 \mathrm{px}, 0.6 \mathrm{px}$ and $1 \mathrm{px}$ ) have been chosen to broadly cover the typical noise found in our experiments (approx. $0.056 \mathrm{px})$.

We start the validation with the convected structures defined in section 3.1.1 and different convecting coefficient values of $C_{\text {conv }}$ : $0,0.1$ and 1 . Figure 2 gives the evolution of the measurement errors versus the ratio between the noise level of the measure, $\sigma_{\text {noise }}$ and the corrected displacement of the measure, $\Delta_{s} \simeq \Delta_{\text {target }}$, where $\Delta_{\text {target }}$ is the user input for the PTVA method. Figure 2 clearly shows a fast increase of the measurements' errors with $\sigma_{\text {noise }} / \Delta_{\text {target }}$. The velocity measurements are more accurate than the acceleration measurements by an order of magnitude. For values of $\sigma_{\text {noise }} / \Delta_{\text {target }}<0.008$, the errors are found to be smaller than $10 \%$ for acceleration (curves ag and ac) and smaller than $0.8 \%$ for velocity (curves vg and vc). When varying the convection intensity, using PTVA, no significant differences are found on the accuracy of the acceleration and velocity. This shows that the method is robust to convection. The increase of the errors with $\sigma_{\text {noise }} / \Delta_{\text {target }}$ is roughly approximated as a power law, so as to represent the sensitivity of the measures to an increase of this ratio. It is found that the acceleration errors increase like $\left(\sigma_{\text {noise }} / \Delta_{\text {target }}\right)^{2.44 \pm 0.05}$ and the velocity errors increase like $\left(\sigma_{\text {noise }} / \Delta_{\text {target }}\right)^{2.06 \pm 0.15}$. This confirms the stronger

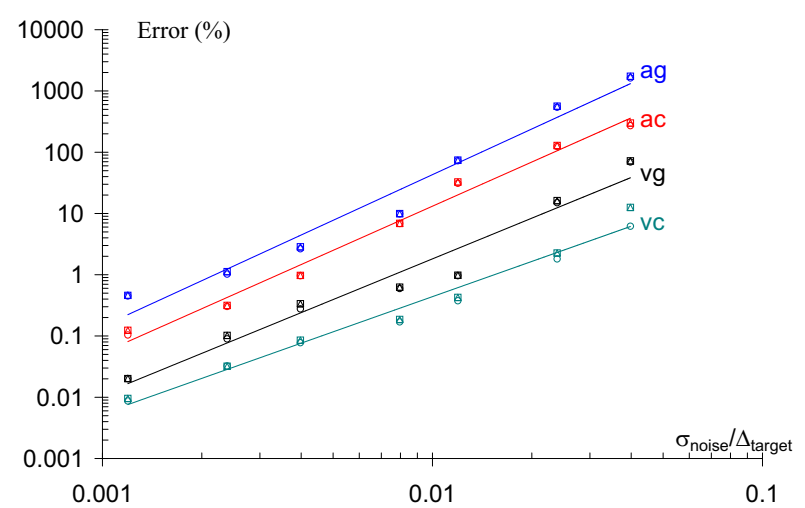

Fig. 2 PTVA's errors on the velocity (vg for Gaussian noise and vc for circular noise) and acceleration (ag for Gaussian noise and ac for circular noise) in function of the ratio $\sigma_{\text {noise }} / \Delta_{\text {target }}$ with $\Delta_{s} \simeq \Delta_{\text {target }}$. The flow structure is steady. The symbols correspond to different cases of convection: ○ for $C_{c o n v}=0, \square$ for $C_{\text {conv }}=0.1, \triangle$ for $C_{c o n v}=1$.

sensitivity to the noise intensity of acceleration measurements compared to velocity measurements.

According to our experimental conditions, with a typical $\sigma_{\text {noise }} / \Delta_{s} \simeq 0.0065$, the errors of the measurements are expected to be about $0.44 \%$ for the velocity and $6.3 \%$ for the acceleration. We do this estimation for the Gaussian noise which appears to be worse than the circular noise in our validation.

We now compare PTVA with methods based on a polynomial approximation with a constant number of positions in the case $C_{\text {conv }}=1$ with $\sigma_{\text {noise }} / \Delta_{s} \simeq 0.0065$ for a Gaussian noise (worst case). To do that, we pay attention to choose the number of positions providing the best accuracy for the non-adaptive methods. ${ }^{4}$ We find errors on velocities about $3 \%$ for $4^{t h}$ order and $2.6 \%$ for $2^{n d}$ order; and errors on acceleration about $25 \%$ for order 4 and $23 \%$ for order 2 . Clearly PTVA is much more accurate: 6 times more accurate on the velocity and 4 times more accurate on the acceleration. Nevertheless, these results show that a relatively low level of noise is important for the quality of the measurement. To obtain a good accuracy, the noise should be kept small compared to the displacement of the particles, and thus small compared to $\Delta_{s}$ and $\Delta_{\text {target }}$.

As a simple convection does not alter significantly the accuracy of the PTVA method, we now introduce a time dependency of the convected geometrical structure presented in section 3.1 .1 by varying $\mathrm{K}$ with time. We use $K(t)=\frac{t}{t_{\max }} \sin \left(2 \pi \frac{t}{\alpha_{T} \Delta t}\right)$, where $\mathrm{t}$ is the time, $t_{\max }$

4 The best accuracy is obtained with 21 positions (order 2 and 4) for the velocity whilst it is obtained with 233 positions (order 4) and 75 positions (order 2) for the acceleration. 9, 21, $47,75,127,181,233,287$ and 339 positions have been tested for the non-adaptive methods. The discrepancy between the best number of positions for the measures of velocity and acceleration could be noted. The average number of positions used by the PTVA algorithm is 123 . The errors obtained for the non-adaptive methods using this number of positions are 19.6 (order 2) and 7.3 (order 4) for the velocity and 23.7 (order 2) and 32.3 (order 4) for the acceleration. 
the duration of the tracking (so as to have $\mathrm{K}$ bounded by \pm 1$), \Delta t$ is the time step of the tracking and $\alpha_{T}$ is the number of tracked positions per period. This leads to periodic changes in the flow structure geometry with an increase in the speed and amplitude of these changes with time. Figure 3 shows errors related to PTVA for the same conditions as figure 2 but with time dependent flow structures obtained with $\alpha_{T}=628$. $\alpha_{T}$ is chosen according to our experimental acquisition frequency. Again, the values of PTVA's errors are not significantly affected by the convection. For $C_{c o n v}=1$ and a Gaussian noise (worst case) with the typical $\sigma_{\text {noise }} / \Delta_{s}$ of the experiments we obtain errors of $2 \%$ for velocity and $15 \%$ for acceleration. When we compare with polynomial approximations, with a constant number of positions (best accuracy case) ${ }^{5}$, we find errors about $16 \%$ for velocity and $48 \%$ for acceleration with $\mathrm{n}=4$ and finally $17 \%$ for velocity and $90 \%$ for acceleration with $n=2$. Even if the time dependency is found to increase the errors of PTVA, it is still much more accurate than non-adaptive methods. It should be noticed that the type of noise and the order of the polynomial interpolation are more important with a time dependent flow structure. Attempting to fit the evolution of the errors with the ratio $\sigma_{\text {noise }} / \Delta_{\text {target }}$ by a power law so as to provide a typical growth of errors with noise, we found that the acceleration errors increase like $\left(\sigma_{\text {noise }} / \Delta_{\text {target }}\right)^{2.5 \pm 0.5}$ and the velocity errors increase like $\left(\sigma_{\text {noise }} / \Delta_{\text {target }}\right)^{1.5 \pm 0.3}$.

To complete the validation of PTVA, we plot in figure 4 the influence of the unsteadiness versus the number of positions per period, $\alpha_{T}$. Varying $\alpha_{T}$ corresponds to vary the frequency of the camera for a given characteristic time scale which corresponds here to one period. When dividing $\alpha_{T}$ by two (from $\alpha_{T}=628$ ), the quality of the velocity and acceleration measurements is not affected as the PTVA algorithm is self-adapting. For divisions of the frequency larger than four $\left(\alpha_{T}<256\right)$, the errors start to grow as power laws of $\alpha_{T}\left(\sim \alpha_{T}^{-0.3}\right.$ for the acceleration and $\sim \alpha_{T}^{-0.48}$ for the velocity). This is due to a reduction of the quality of the temporal sampling compared to the strong unsteadiness of the flow. Nevertheless, the fact that the PTVA method stays relatively robust for a broad range of "sampling frequencies" (more than one decade, $\left.39 \leq \alpha_{T} \leq 628\right)$ is noticeable.

This extra accuracy has a cost as the PTVA computations are about 6.4 times longer than methods using a constant number of positions (speed test performed on

\footnotetext{
5 The best accuracy is obtained with 153 positions for the velocity and 199 positions for acceleration using order 4 , whilst it is obtained with 35 positions for velocity and with 153 positions for acceleration using order 2. 17, 35, 63, 93, 153, 199, 243, 289 and 335 positions have been tested for the non-adaptive methods. The average number of positions used by the PTVA algorithm is 115 . The errors obtained for the non-adaptive methods using this number of positions are 61.3 (order 2) and 24.2 (order 4) for the velocity and 93.1 (order 2 ) and 53.2 (order 4) for the acceleration.
}

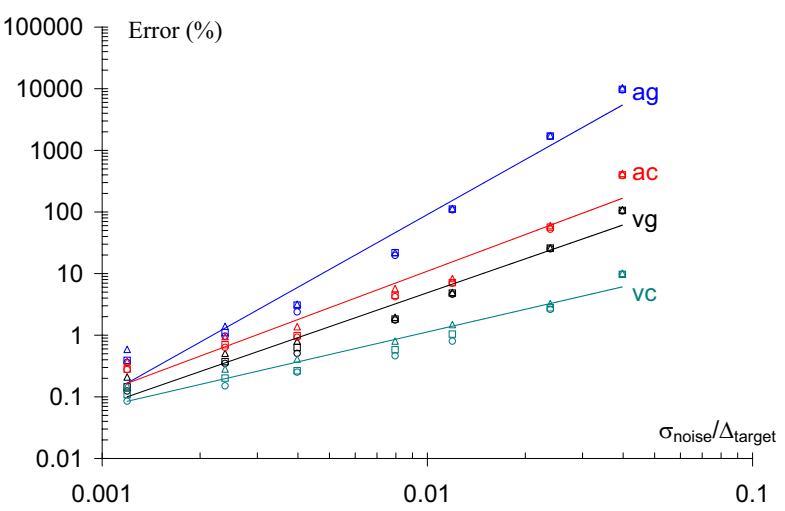

Fig. 3 PTVA's errors on the velocity (vg for Gaussian noise and vc for circular noise) and acceleration (ag for Gaussian noise and ac for circular noise) in function of the ratio $\sigma_{\text {noise }} / \Delta_{\text {target }}$ with $\Delta_{s} \simeq \Delta_{\text {target }}$. The flow structure is unsteady, $\alpha_{T}=628$. The symbols corresponds to different cases of convection: $\circ$ for $C_{\text {conv }}=0, \square$ for $C_{c o n v}=0.1, \triangle$ for $C_{\text {conv }}=1$.

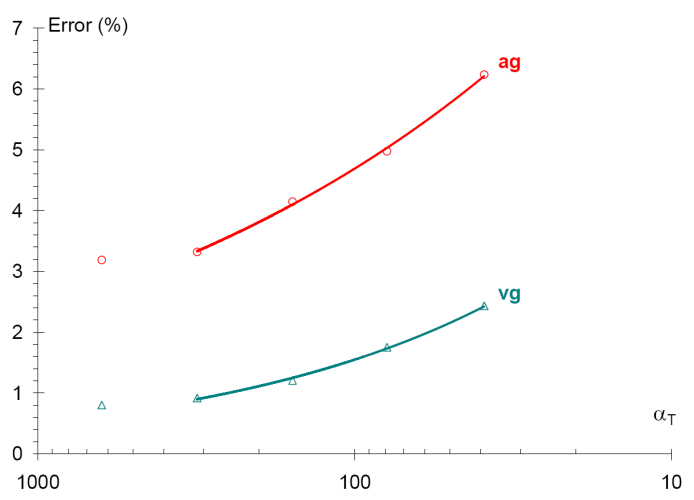

Fig. 4 PTVA's errors on the velocity (vg, Gaussian noise) and acceleration (ag, Gaussian noise) for various sampling (unsteadiness) coefficient $\alpha_{T}$ while keeping the characteristic noise and convection constant: $\sigma_{\text {noise }} / \Delta_{\text {target }} \simeq 0.004\left(\Delta_{s} \simeq\right.$ $\Delta_{\text {target }}$ ) and $C_{\text {conv }}=1$. The lines correspond to power law fits.

a computer with an Intel Pentium 4 at $3.00 \mathrm{GHz}$ with 1.00 GB ram).

It should be noted that if the PTVA algorithm is stopped after the first stage, the errors obtained are about two times larger than the ones obtained with the complete algorithm. This highlights the importance of the mean Lagrangian velocity removal stage in the PTVA method.

\section{Experiments}

\subsection{Brief Description of the rig and forcing}

We use the same experimental facility as Rossi et al. (2006a), where a horizontal shallow layer of brine (salt water, $158 \mathrm{~g} / 1 \mathrm{NaCl}$ ), is forced by a fractal distribution of opposite pairs of Lorentz forces. These electromagnetic (EM) forces are generated by an electric current 


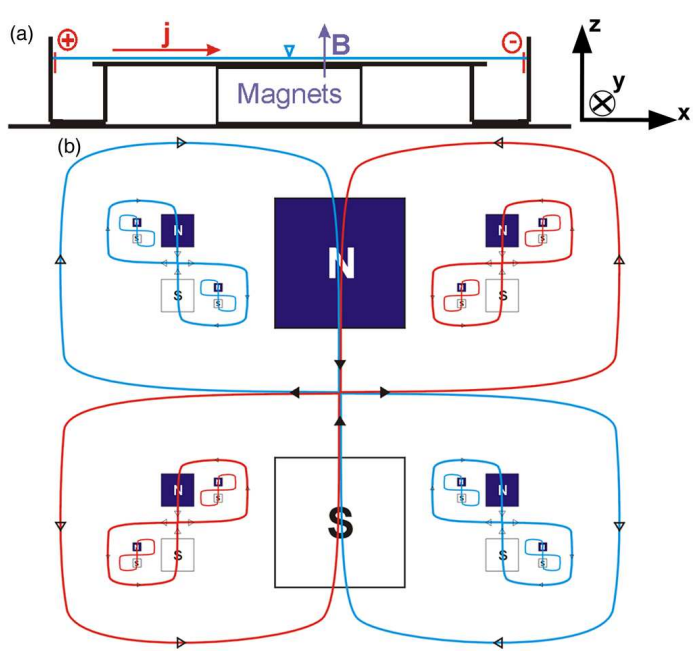

Fig. 5 (a) Rig's schematic for electromagnetic forcing of a shallow brine layer. (b) Schematic of the permanent magnets placed under the brine supporting wall and of a cat's eyes within cat's eyes flow structure.

through the brine and permanent magnets of various horizontal sizes $(10 \mathrm{~mm}, 40 \mathrm{~mm}, 160 \mathrm{~mm})$, placed under the bottom wall which supports the brine. Figure 5a gives a schematic of the rig and figure $5 \mathrm{~b}$ a top view of the distribution of permanent magnets (North and South) placed under the wall. The thickness of the shallow brine layer is about $\mathrm{H}=5 \mathrm{~mm}\left(H_{\text {mean }}=5.009 \pm\right.$ $0.121 \mathrm{~mm}$ ). The quasi-two-dimensionality of the flow has been checked and verified. These EM forces generate a multi-scale laminar flow that we illustrate here with the particles trajectories given in figure 6 . One advantage of the present flow is that we know and control its geometry, topology and time dependency. For more details, see Rossi et al. (2006a).

We use Image Analysis techniques to perform measurements on this flow. The experimental setup consists in a 2 ADC high definition camera (2048x2048 pixel $^{2}$ for a maximum acquisition frequency of $14 \mathrm{~Hz}$ and a 14 bit depth), placed orthogonally to the measurement plane and by two $500 \mathrm{~W}$ lamps that light up the investigation field. The flow is seeded with particles of Chemigum P83, having a density of 1.03 compared to fresh water. The size of particles on filmed images is about $5 \pm 2.5$ pixels. The data used are filmed with an acquisition frequency of $10 \mathrm{~Hz}$. Two frames of different sizes $(1015.4 \mathrm{~mm}$ and $842.1 \mathrm{~mm}$ ) are investigated, so as to measure the entire flow and to increase the resolution at the small lengthscales.

For this steady forcing case $(\mathrm{I}=0.53 \mathrm{~A})$ we have performed 108 runs with the large frame and 22 runs with the small frame, each run being of about 1000 pictures. This corresponds to an acquisition time of $100 \mathrm{~s}$, which is much longer (about 5 times) than the turnover time of the integral length-scale $(\simeq 19.2 s)$, Rossi et al.(2006a). On Figure 6 (and on following results), only trajecto-
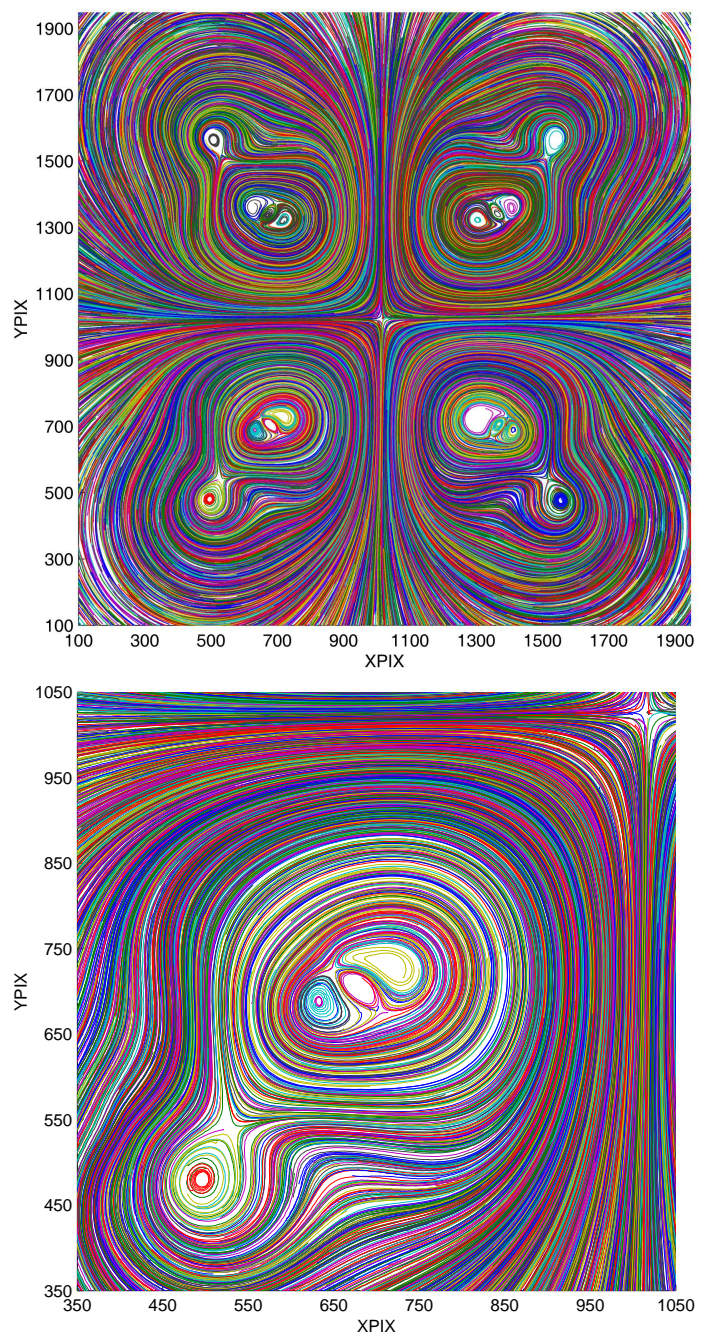

Fig. 6 Particle tracking, full flow field (top) and zoom on bottom left quarter.

ries tracked for at least one turnover time are taken into account.

\subsection{PTVA parameters}

To measure the velocity and acceleration we use the PTVA method with $\Delta_{\text {target }}=4 \pi \mathrm{mm}$ as input. This is equivalent to $\Delta_{\text {target }} \simeq 25 p x$ in the large frame and $\Delta_{\text {target }} \simeq 31 p x$ in the small one. The corresponding smallest diameter that the method can track is about $\phi_{\text {small }} \simeq 2.7 \mathrm{~mm}$. $\phi_{\text {small }}$ is much smaller than the small magnet size $(10 \mathrm{~mm})$ and smaller than the smallest eddy effectively tracked ( $5 \mathrm{~mm}$ of diameter), so the method does not introduce an artificial smoothing even at the small scales of the flow. In addition, the minimum and maximum number of positions used by the PTVA algorithm are respectively set to 11 and 267 for these experiments with $\Delta_{\min }=11 p x$ and $\Delta_{\max }=60 p x$. 

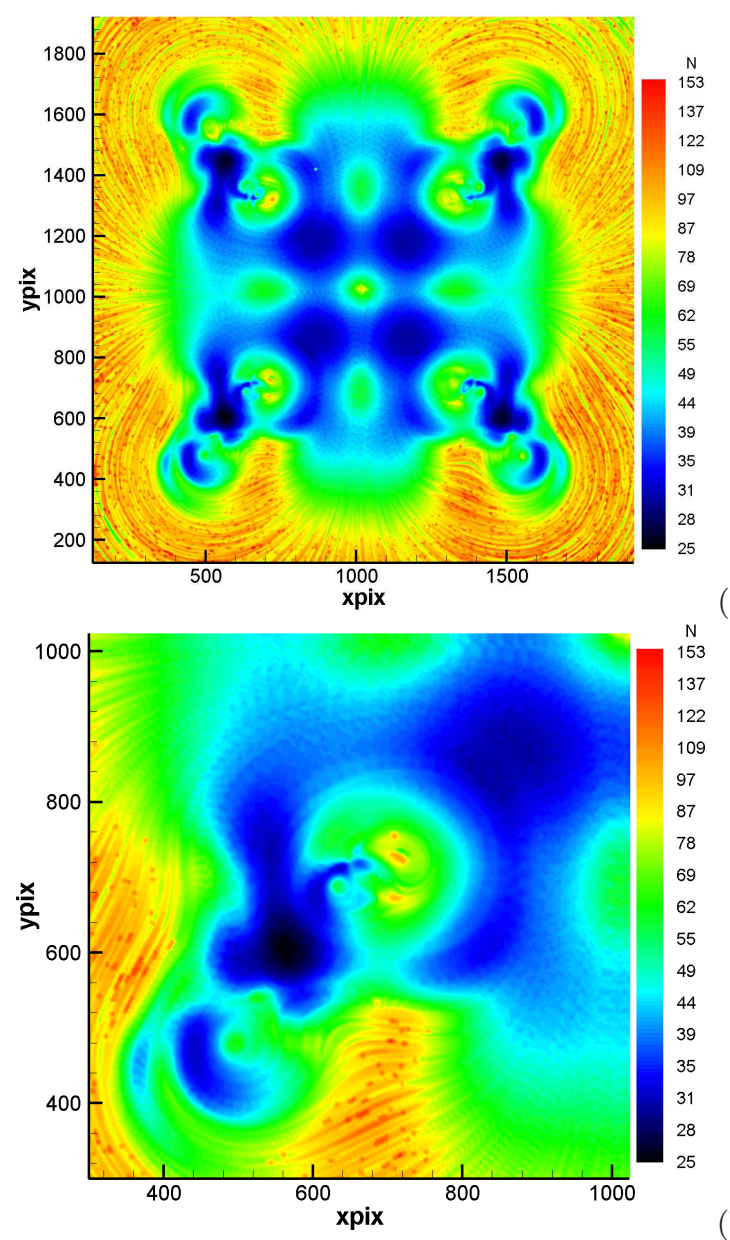

(a)

Fig. 7 Spatial distribution of the number of positions, N used by PTVA method: (a) large frame, (b) local zoom of (a).

\section{Comparison of PTVA versus methods using a constant number of positions on experimental data}

Before comparing the PTVA and the methods using a constant number of positions on selected trajectories, we illustrate the variation of the number of positions used by PTVA. Figure 7, clearly shows that the PTVA algorithm really adapts to the local properties of the flow. In fact, the comparison between figure 7 and 10 shows that $\mathrm{N}$ adapts to the intensity of the acceleration so as to accurately measure high and low values of acceleration at all length-scales.

To briefly examine and illustrate the differences on the acceleration measurements obtained on our flow with PTVA and with other non-adaptive methods (e.g. Virant and Dracos (1997), La Porta et al. (2001), Voth et al. (2002), Luthi et al. (2005), Mordant et al. (2004)), selected trajectories extracted by PTV on our flow are given as input. In particular, two methods based on a moving polynomial approximation (of order 2 and 4, respectively) with a fixed number of positions (25) have been chosen, in order to show that the increase in accuracy of PTVA is not only due to the higher order of the polynomial approximation. The smallest turn-over time scale of the flow, $T_{\text {small }}$, is about $5 \mathrm{~s}$. With a frequency of $10 \mathrm{~Hz}$, this leads to a maximal number of positions of about 25 to have the duration of the tracked positions used for a polynomial approximation smaller than $T_{\text {small }} / 2$. In addition, it could be noticed that this value is one of the local maximum for the probability distribution of the number of positions used by the PTVA method.

The selected trajectories for this comparison are shown on figure $8 \mathrm{~d}$, with a red asterisk marking the initial position and a black circle the final one. These trajectories have been chosen as a "synthesis" of the flow: in fact, the black one (which crosses the North big magnet and so experiences the highest velocities in the flow, while traveling along an almost straight line, and goes close to the large scale hyperbolic stagnation point) and the blue one are typical of the large scales of the flow, the red one (that goes close to a medium scale hyperbolic stagnation point and then describes an eight of the same scale) is representative of the medium scales, whereas the green one (that travels close to both elliptical and hyperbolic stagnation points of the small scales) identifies some features of the small scales. Figures $8(\mathrm{a}, \mathrm{b}, \mathrm{c})$ give the comparison of the $\mathrm{x}$ component of acceleration measured by different methods for the trajectories of figure $8 \mathrm{~d}$ (same colors). PTVA is amenable to provide an accurate measure of the acceleration, without deleting small fluctuations and without the outliers that arise from the other two methods (see green, red and magenta trajectories). It is also noticeable that PTVA is able to measure a sudden change in the acceleration sign and thus shows a good sensitivity to inflection points, as illustrated by the blue curve.

\section{Results}

\subsection{Eulerian fields}

The Eulerian repeatability of our experiment, combined with the large number of trajectories and positions, allows us to extract the Eulerian information all over the investigation field from the Lagrangian measurements. This permits us not only to extract the velocity field but also the acceleration field. A good accuracy on these two fields allows the decomposition and analysis of the components of Navier-Stokes equation, $\mathbf{a}=\frac{\partial \mathbf{u}}{\partial \mathbf{t}}+(\mathbf{u} \cdot \nabla) \mathbf{u}=$ $-\frac{1}{\rho} \nabla \mathbf{P}+\nu \nabla^{2} \mathbf{u}+\mathbf{f}$, where $\mathrm{P}$ is the pressure, $\rho$ the fluid density, $\nu$ the fluid kinematic viscosity and $\mathbf{f}$ the forcing.

The method used to construct the grid is adaptive: we adjust the size of the extrapolation windows depending on the number of positions inside these windows. When 

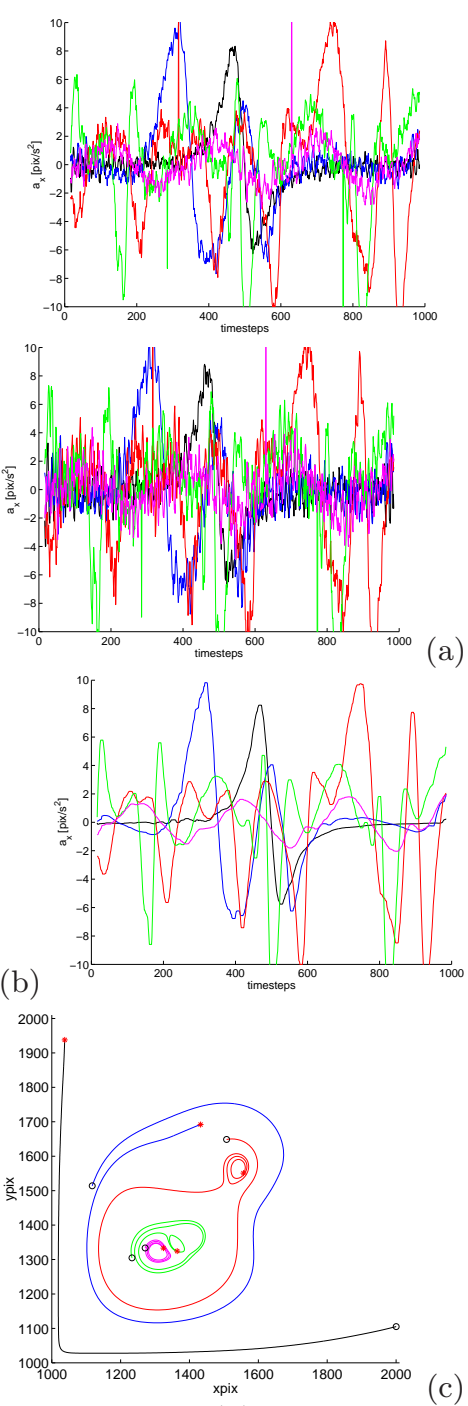

(d)

Fig. 8 Comparison of the measured x-component of the acceleration in $p i x / s^{2}$, along the trajectories of figure d: moving polynomial approximation with constant number of positions of order 2 (a) and order 4 (b), PTVA (c). $1 \mathrm{~mm}$ is about 2 pixels.

the extrapolation windows are significantly smaller than the size of small magnets, it is based on Smooth Particle Hydrodynamic approximations, Monaghan (1992), (with more than 18 positions within the extrapolation windows). When the extrapolation windows are of the order or larger than the size of the small magnets (very rare cases), it is based on a bi-quadratic approximation (with feedback on the standard deviation and more than 24 positions within the extrapolation windows). With more than $2.610^{6}$ positions for Lagrangian velocity and acceleration measurements, we extract the grids with a mesh'size of 3 pixels while the extrapolation windows are typically about 6.75 pixels. These extrapolation windows are significantly smaller than the size of the small magnets (20 pixels). Taking advantage of a $55 \%$ overlap of the constructed grids, most of the results presented in this section include a $3 \times 3$ median smoothing.

Figure 9 gives the velocity field extracted from PTVA data. This illustrates the multi-scale flow generated by the forcing given in figure 5. The highest velocities are found above the two biggest magnets. The stagnation point connected to the large scales of the flow is clearly noticeable as the spot with zero velocity values in the center of the field, figure 9a. The flow is well defined at all scales, as shown in figures $9 \mathrm{~b}$ and 9c. On theses figures, the red circles refer to elliptical stagnation points whilst the red squares refer to hyperbolic stagnation points. This velocity field constructed from PTVA data is the same as the one obtained by Rossi et al. (2006a) (forcing $\mathrm{I}=0.53 \mathrm{~A}$ ) with Particle Image Velocimetry (PIV), except that here the resolution is higher than the one of PIV fields (600x600 versus $287 \times 287)$ and the extrapolation windows' size is typically 2.4 times smaller than the PIV correlation windows' size.

The Eulerian acceleration field, along with some acceleration lines, is shown on figure 10. Regarding the spatial distribution of acceleration intensities, the highest acceleration values arise where both strain and velocity are high. This occurs in the vicinity of the hyperbolic stagnation point of the medium scales. For a steady flow, the critical elliptical and hyperbolic points in the velocity field are also zero acceleration points. At all flow scales, the acceleration field also shows some zero acceleration points which are not velocity stagnation points (e.g. above the two biggest magnets). The red circles and squares refer to elliptic and hyperbolic velocity stagnation points. In fact, in the case of zero acceleration points arising from velocity stagnation points, the acceleration lines connected to them have all the "same direction": they go towards the elliptical stagnation points, leading to locally high negative values of $\nabla \cdot \mathbf{a}$, and outwards from the hyperbolic ones, leading to locally high positive values of $\nabla \cdot \mathbf{a}$, see figure 11 . The zero acceleration points belonging only to the acceleration field display both acceleration lines going toward and away from them; they correspond to local low absolute values of $\nabla \cdot \mathbf{a}$, see figure 11.

Moreover, figure 10 reveals a multi-scale distribution of acceleration "sources" and "sinks" emanating from zero acceleration points which are also extremum for $\nabla$. a. The scale of these acceleration "sources" and "sinks" can be related to the length of the acceleration lines emanating from them, similarly to the multi-scale distribution of stagnation points, e.g. Rossi et al. (2006b). It should be noticed here that the acceleration lines emanating from "sources" (respectively "sinks") can be directly connected to "sinks" (respectively "sources") of different scales. This clearly works differently than streamlines, where elliptical stagnation points are not directly connected to hyperbolic stagnation points via streamlines. Such information contained in the acceleration field is thus extremely complementary to the velocity field as 
(a)
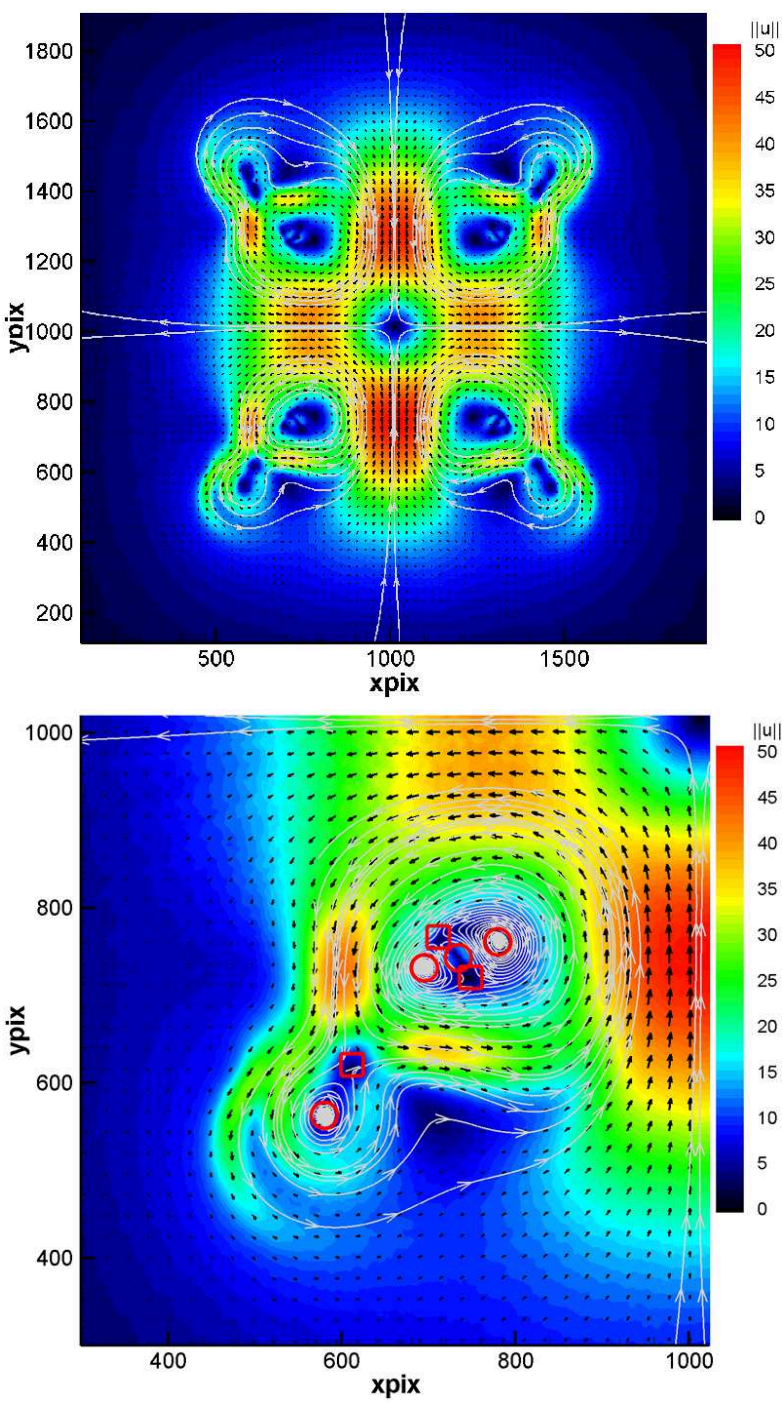

(b)

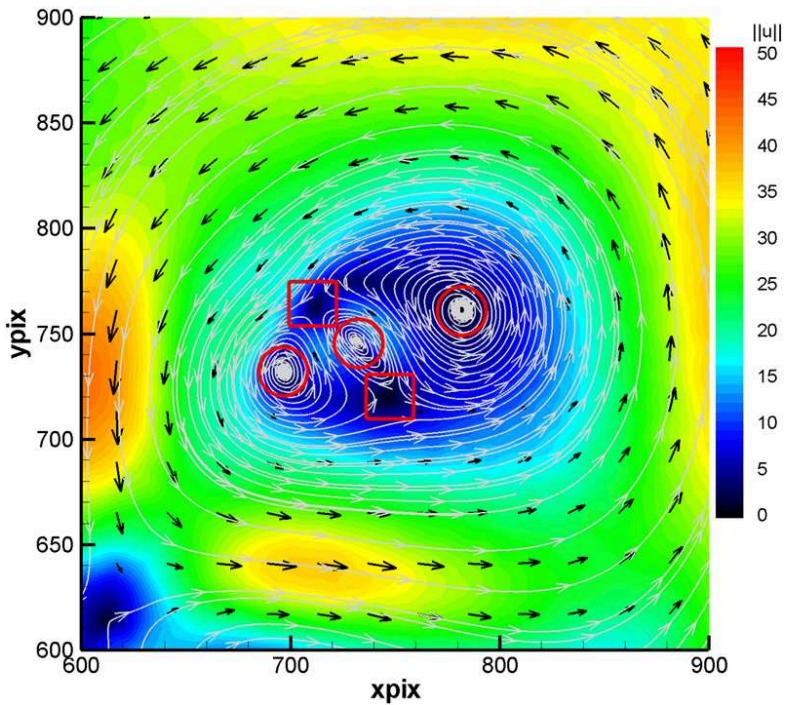

(c)

xpix

Fig. 9 Velocity field: the velocity intensity $\|\mathbf{u}\|$ is in pix/s, 1 arrow for 64 are plotted, lines correspond to streamlines. $1 \mathrm{~mm}$ is about 2 pixels and the mesh is of $600 \times 600$ points with a size of the mesh of 3 pixels. (a) large frame; (b) zoom of (a); (c) zoom of (b). it highlights which flow structure is connected to which ones and so draws a "web of the flow". We expect such information to be important for flow control applications.

Figure 12 gives the field of Navier-Stokes equation's viscous term, $\nu \nabla^{2} \mathbf{u}$, computed from the velocity. As it implies a second order spatial derivative, it is very sensible to the noise and the quality of the measurement. Despite that, the measured velocity field is good enough to allow the computation of the velocity Laplacian. The rms of the viscous term $\left(0.13\right.$ pixs $\left.^{-2}\right)$ is about 40 times smaller than the rms of the acceleration. To illustrate the coherence of the viscous term measurements, figure $12 \mathrm{~b}$ gives a zoom of figure 12a with some pointed areas: the red circle refers to the center of an eddy, the red square refers to a velocity hyperbolic stagnation point and the red triangle refers to a region of shear flow. Where the velocity vectors (figure $9 \mathrm{~b}$ ) and the viscous term vectors (figure $12 \mathrm{~b}$ ) have an opposite direction, the viscous term is subtracting energy from the flow ("pulling it") and where they have the same direction the viscosity is "pushing" the flow. As the velocity vectors and viscous term around the elliptical stagnation point have opposite direction, the viscosity is acting against the rotation of the eddy. On the left of the triangle, a tongue of faster fluid squeezes in a zone of slower fluid: so the viscosity acts to slow it down. Over the triangle, on the tongue, the viscous term acts in the same direction as the velocity as this "small scale low speed area" bridges two "higher speed areas", see figure $9 \mathrm{~b}$.

As the forcing is known with significant values of the electromagnetic forces concentrated only above the magnets (see figure 5), which are also of weak percentage area $(2.8 \%)$, the pressure gradient field, which is difficult to measure experimentally, can be extracted from the difference between acceleration and viscous terms in Navier Stokes equation. In fact the viscous term is small compared to the pressure term for the vast majority of the flow. In the case where $\mathbf{a}=-\frac{1}{\rho} \nabla \mathbf{P}$, all the acceleration lines converging towards a node (zero acceleration point) correspond to a decreasing pressure towards this point, hence being a local minimum of pressure ("eddy center"). Similarly, all the acceleration lines diverging from a node (zero acceleration point) correspond to an increasing pressure towards this point, which is a local maximum of pressure (hyperbolic stagnation point, see figure 10).

To analyse the relation acceleration-velocity as well as the power input/output in the flow, figure 13 gives the scalar product $\mathbf{u} \cdot \mathbf{a}$ which is computed on PTVA data before constructing its Eulerian field. $\mathbf{u} \cdot \mathbf{a}$ is proportional to the tangential acceleration, hence it shows where fluid particles increase their velocity (velocity and acceleration in the same direction) or decrease their velocity (acceleration and velocity in opposite directions). This scalar product also gives the power input and output in the flow per unit mass. In this flow the main power input comes from the forcing above the magnets, it is then coher- 


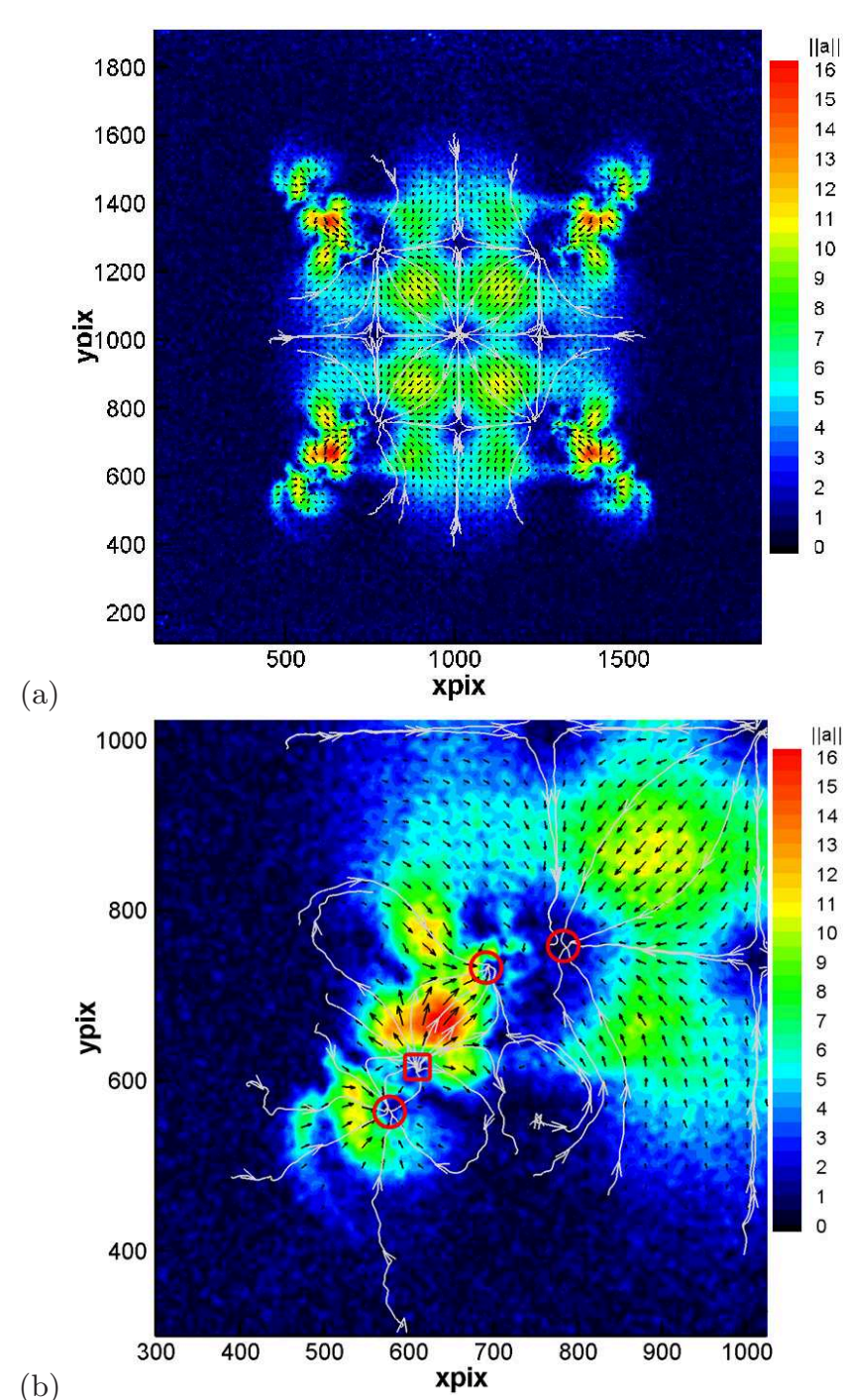

(b)

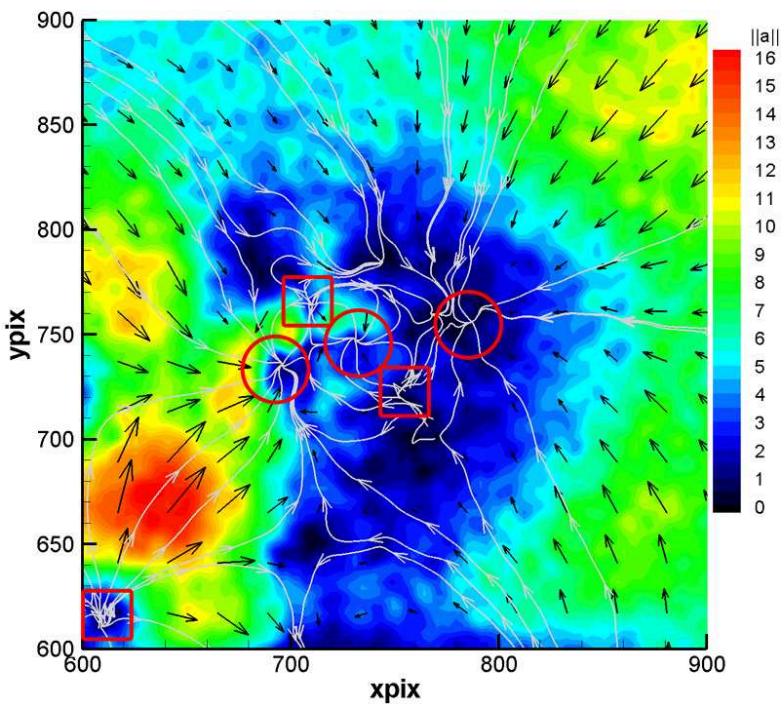

(a)

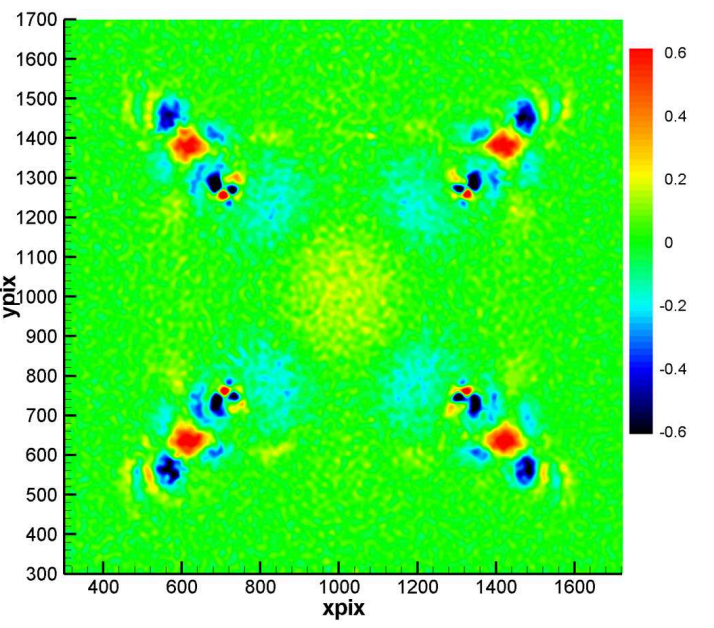

Fig. 11 Divergence of acceleration in $s^{-2}$; (the color map does not used full scale which is about \pm 1 to keep values above the large scale stagnation point visible).
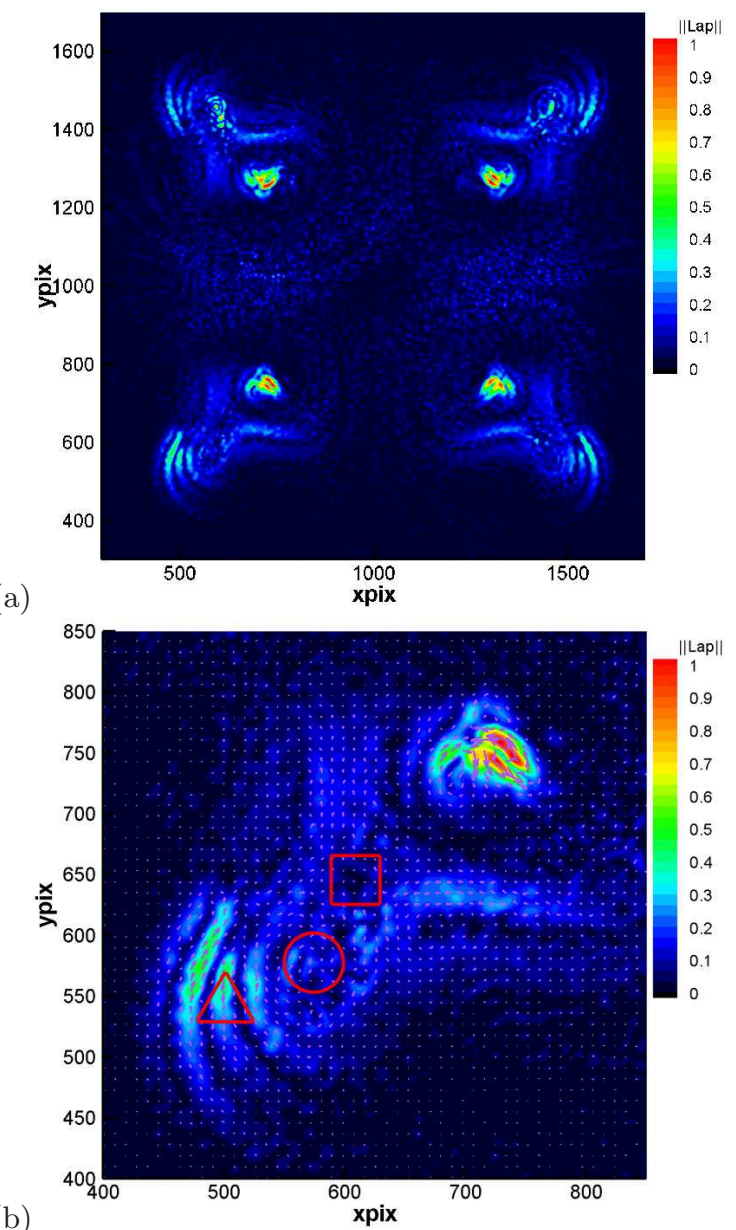

Fig. 12 Viscous Term, $\nu \nabla^{2} \mathbf{u}$, computed from the velocity field. (b) is a zoom of (a). The square indicates the region of medium scale hyperbolic stagnation points. The circle notes the region of an eddy. The triangle shows the region of a low speed flow region in between two higher speed regions. See figure $9 \mathrm{~b}$ for comparison. The color scale corresponds to $\left\|\nu \nabla^{2} \mathbf{u}\right\|$ and is in pixs ${ }^{-2}$.

Fig. 10 Acceleration field: the acceleration intensity $\mid \mathbf{a}$ in $p i x / s^{2}, 1$ arrow for 64 are plotted, lines correspond to lines parallel to acceleration vectors, $1 \mathrm{~mm}$ is about 2 pixels, the mesh is of $600 \times 600$ points with a mesh's size of 3 pixels. (a) large frame; (b) zoom of (a); zoom of (b). 


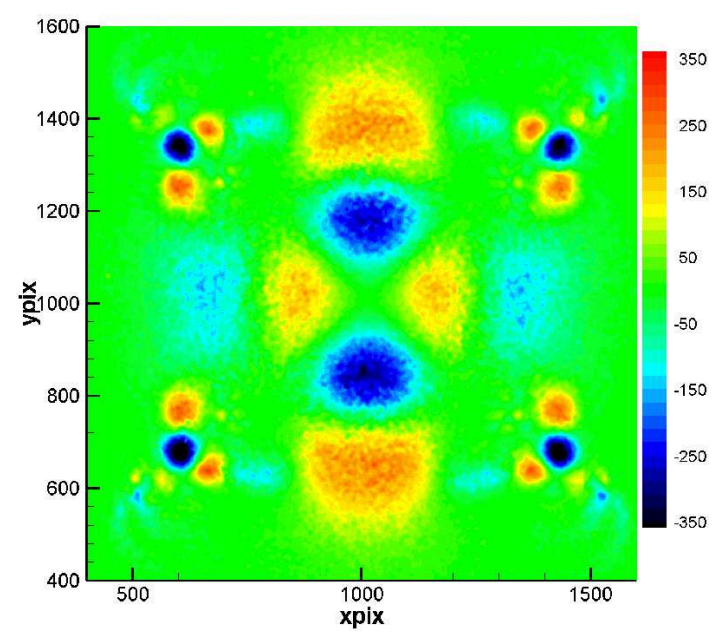

Fig. 13 Scalar product, $\mathbf{u} \cdot \mathbf{a}$, in $p i x^{2} s^{-3}$.

ent that $\mathbf{u} \cdot \mathbf{a}$ identifies the magnets' positions (compare with figure 5). In addition, $\mathbf{u} \cdot \mathbf{a}$ also illustrates the power input/ouput related to the work of pressure terms. For example the alternated values of $\mathbf{u} \cdot \mathbf{a}$ surrounding the large scale stagnation point are governed by the work of pressure terms around this point.

\subsection{PDF of acceleration}

The probability distribution function (PDF) of $\mathrm{x}$-compone (black circles) and y-components (red asterisks) of the acceleration, normalized with the root mean square acceleration $a_{r m s}$ of the flow (6.304 pixel $/ \mathrm{s}^{2}$ corresponding to $3.12 \mathrm{~mm} / \mathrm{s}^{2}$ ), are plotted on figure 14 . Also plotted on figure 14 is the Gaussian curve that best-fits the data in a least mean square sense (dashed blue line) and the empirical function proposed by Voth et al. (2002): $P(a)=$ $C \exp \left(-\left[a^{2}\right] /\left[\left(1+|a \beta / \sigma|^{\gamma}\right) \sigma^{2}\right]\right)$ (solid green line). Both acceleration components are Gaussian for more than one decade of the PDF (and absolute values of acceleration components smaller than $1.5 a_{r m s}$ ) and then exhibit larger tails. The fitting parameters turn out to be similar between the two experiments: $\beta=0.5621, \sigma=0.6143$ and $\gamma=1.266$ in the present case, and $\beta=0.539$, $\sigma=0.508, \gamma=1.588$ in Voth et al. (2002).

The shape of the PDF of this multi-scale flow is very similar to the one usually encountered in turbulence, e.g. La Porta et al. (2001), Voth et al. (2002). It is then very tempting to attribute this statistic to a new turbulentlike feature of this multi-scale flow whilst keeping in mind that this flow is still laminar and quasi-steady.

\section{Conclusions}

We have presented and validated a new method to measure velocity and acceleration from the processing of par-

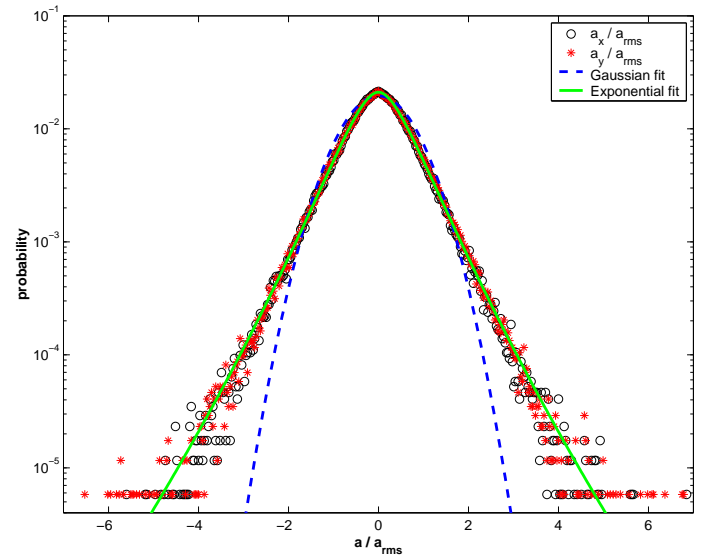

Fig. 14 PDF of the two acceleration components, $a_{x}$ and $a_{y}$, normalized by the acceleration $\mathrm{rms}\left(6.304 \mathrm{pix} / \mathrm{s}^{2}\right), 1$ pixel is about $0.5 \mathrm{~mm}$.

ticle tracking data. The method self-adapts to the local flow properties. It has been shown that PTVA allows to increase the quality of measurements compare to methods using a constant number of positions. Errors are about 6 times smaller for velocity and about 4 times smaller for acceleration. PTVA has also been shown to be robust to convection and time dependency. As it does not involve any complex experimental set-up, PTVA can be a useful tool for our future measurements and for other researchers working in the field. Moreover, as it takes as input the particles' positions extracted from nts TV, it can be used to improve the quality of previous PTV measurements.

We apply this PTVA method to a controlled multiscale flow generated by multi-scale electromagnetic forcing. The non trivial construction of various Eulerian fields, e.g. acceleration field, provides deeper insights in the flow structure. We observe a multi-scale distribution of "sources" and "sinks" of acceleration which are related to local maxima and minima of pressure. These sources and sinks are connected via lines parallel to the acceleration vectors defining a "web structure" of the flow complementary to the velocity field structure. The flow's power input/output (estimated by $\mathbf{u} \cdot \mathbf{a}$ ) highlights the work of electromagnetic forces and pressure terms. Finally, the measurements have shown that the acceleration PDF of this turbulent-like flow presents strong similarities with the one obtained in highly turbulent flows. Investigation of this latest point and additional quantifications of acceleration fields properties are kept for future research.

To conclude, it should be noticed that the two key points of the method, mean Lagrangian velocity correction and adaptability, have a larger impact on the quality of the acceleration measurement than the order of the polynomial function used to locally approximate the trajectories. In addition, the PTVA method does not require the existence of eddies to work correctly and thus the PTVA algorithm could be applied to a broader range of flows than the present experiments. 
We acknowledge the Marie Curie Multi-Partner European Training Site on Environmental Turbulence, the Royal Society, The Leverhulme Trust, the EPSRC, S. Dalziel and Digiflow's group, G. Querzoli and J.C. Vassilicos.

\section{References}

1. Biferale, L., Boffeta, G., Celani, A., Lanotte, A., Toschi, F. "Lagrangian statistcs in fully developed turbulence", arXiv:nlin.CD/0402032, 1-4 (2004)

2. Chen, L., Goto, S., Vassilicos, J.C. "Turbulent clustering of stagnation points and inertial particles", J. Fluid Mech. 55 143-154 (2006)

3. Christensen, K.T., Adrian, R.J. "Measurement of instantaneous eulerian acceleration fields by particle image accelerometry: method and accuracy", Exp. Fluids , 33, 759769 (2002)

4. Dalziel, S.B., "Decay of rotating turbulence: some particle tracking experiments", Appl. Scien. Res., 49, 217-244 (1992)

5. Dong, P., Hsu, T.Y., Atsavapranee, P., Wei T. "Digital particle image accelerometry", Exp. Fluids , 33, 759-769 (2002)

6. Goto, S. , Osborne, D.R., Vassilicos, J.C., Haigh, J.D. "Acceleration statistic as measures of statistical persistence of streamlines in isotropic turbulence", Phys. Rev. E., 71, 015301(R)(2005)

7. La Porta, A., Voth, G. A., Crawford, A. M., Alexander, J., Bodenschatz, E., "Fluid particle accelerations in fully developed turbulence", Nature, 409, 1017-1019 (2001)

8. Lowe, K.T., Simpson R.L., "Measurements of velocity acceleration statistics in turbulent boundary layers" TSFP, 4, 1043-1048 (2005)

9. Luthi, B., Tsinober, A., Kinselbach, W., "Lagrangian measurement of vorticity dynamics in turbulent flows", J. Fluid. Mech., 529, 87-118 (2005)

10. Monaghan, J.J. "Smoothed particle hydrodynamics", Annu. Rev. Astron. Astrophys., 30, 543-574 (1992)

11. Mordant, N., Crawford, A.M., Bodenschatz, E., "Experimental Lagrangian acceleration probability density function measurement", Phisica D, 193, 245-251 (2004).

12. Ottino,J.M., "The kinematics of mixing: stretching, chaos, and transport", 1-364, Cambridge University Press, (1989)

13. Querzoli, G., "A Lagrangian study of particle dispersion in the unstable boundary layer", Atmospheric Environment, vol 30(16) 2821-2829 (1996)

14. Rossi, L., Hascoet, E. Vassilicos, J.C., Hardalupas, Y. "2D fractal flow generated by electromagnetic forcing: laboratory experiments and numerical simulations", TSFP, 4, 485-490 (2005)

15. Rossi, L., Vassilicos, J.C., Hardalupas, Y. "Electromagnetically controlled multiple scale flows", J. Fluid Mech. 558, 207 - 242, (2006a)

16. Rossi, L., Vassilicos, J.C., Hardalupas, Y. "Multi-scale laminar flows with turbulent-like properties", Phys. Rev. Lett. 97, 144501 (2006b)

17. Tsinober, A., "An informal introduction to turbulence", 1-324. Kluwer Academic Publishers, (2001a)

18. Tsinober, A., Vedula, P., Yeung, P.K., "Random Taylor hypothesis and the behavior of local and convective accelerations in isotropic turbulence", Phys. Fluids (13)7, 19741984 (2001b)

19. Vassilicos, J.C., "Mixing in vortical, chaotic and turbulent flows", Phil. Trans. R. Soc. Lond. A, 360, 2819-2837 (2002)
20. Vedula, P., Yeung, P.K., "Similarity scaling of acceleration and pressure statistic in numerical simulations of isotropic turbulence", Phys. Fluids, (11)5, 1208-1220 (1999)

21. Virant, M., Dracos, T., "3D PTV and its application on Lagrangian motion", Meas. Sci. Technol, 8, 1539-1552 (1997)

22. Voth, G. A., La Porta, A., Crawford, A. M., Alexander, J., Bodenschatz, E., "Measurement of particle accelerations in fully developed turbulence", J. Fluid. Mech., 469, 121$160(2002)$ 